\title{
La verve musicale de Vladimir Jankélévitch
}

\section{Claude Coste}

\section{OpenEdition}

Journals

Édition électronique

URL : https://journals.openedition.org/recherchestravaux/722

DOI : 10.4000/recherchestravaux.722

ISSN : 1969-6434

\section{Éditeur}

UGA Éditions/Université Grenoble Alpes

\section{Édition imprimée}

Date de publication : 15 décembre 2014

Pagination : 189-203

ISBN : 978-2-84310-291-2

ISSN : 0151-1874

\section{Référence électronique}

Claude Coste, "La verve musicale de Vladimir Jankélévitch », Recherches \& Travaux [En ligne], 85 | 2014, mis en ligne le 15 juin 2016, consulté le 29 octobre 2021. URL : http://journals.openedition.org/ recherchestravaux/722 ; DOI : https://doi.org/10.4000/recherchestravaux.722 
Claude Coste

Univ. Grenoble Alpes

\section{La verve musicale de Vladimir Jankélévitch}

La musique a partie liée avec l'ineffable, qui met en branle l'état de verve en l'homme et fait naître une espérance lumineuse $\mathrm{I}^{\mathrm{I}}$.

Dans Le Plaisir du texte, Roland Barthes présente la peur comme un «sentiment médiocrement indigne ${ }^{2}$ ", qu'aucune philosophie, qu'aucune pensée de la modernité n'acceptent de prendre en charge. Dans un tout autre domaine, la verve ne passerait-elle pas pour une qualité "médiocrement digne»? De la verve, on n'entend jamais dire de mal. Parler avec verve, animer une soirée ou un dîner par la verve de son esprit, tenir une assemblée en haleine par la verve de son discours : autant d'exemples positifs (on en trouverait bien d'autres) qu'aucune critique ne vient jamais ternir. Pourtant, comparée à l'intelligence, au style, à l'art, à l'inspiration, la verve occupe une place toujours secondaire, comme la peur ne mérite jamais, selon Barthes, de condamnation franche et définitive. Pour rehausser la verve, il faut la considérer comme l'antichambre de l'enthousiasme, y voir le symptôme par lequel le dieu se manifeste en l'homme qu'il inspire et fait parler : il faut lire dans la verve les signes extérieurs de l'esprit au travail. Prise pour elle-même, la verve a des admirateurs, mais jamais de fidèles et encore moins de martyrs.

Il revenait à Vladimir Jankélévitch de relever le défi que l'insignifiance lance à la philosophie. À une époque, l'après-guerre, où la pensée se donnait des objets plus ambitieux - l'Histoire, la structure, la lutte des classes -, l'auteur de L'Ironie, du Je-ne-sais-quoi et Le Presque-rien place la verve au cour de sa réflexion philosophique, n'hésitant pas à faire d'une qualité médiocre un

I. F. Schwab préface à Liszt. Rhapsodie et improvisation, Paris, Flammarion, 1998, p. 2I.

2. R. Barthes, Le Plaisir du texte, Paris, Seuil, 1973, p. 249. 
concept fondamental pour comprendre le fonctionnement de la condition humaine. Qui sait que Jankélévitch vouait un culte à Flers et Caillavet, qu'il riait aux larmes en entendant les répliques pleines de verve du Roi ou de L'Habit vert? On connaît davantage sa passion pour la musique, ses ouvrages consacrés à Fauré, Ravel, Debussy, et sa grande admiration pour le génie compositionnel de Liszt. Pour lui, musique et philosophie posent les mêmes questions fondamentales sur la verve et sur le monde.

Voilée par le brouillage des rééditions, la bibliographie mérite quelques éclaircissements. En 1955, chez Flammarion, paraît Rhapsodie et improvisation musicale, un ouvrage qui ne sera jamais réédité; en 1979, Plon publie un Essai sur la virtuosité, première partie d'un diptyque à venir intitulé Liszt et la rhapsodie $e^{3}$ La seconde partie, annoncée et jamais réalisée, aurait sans doute consisté en un développement des chapitres I et $\mathrm{v}$ du livre de 1955. Ce sont ces deux chapitres que Françoise Schwab réédite en 1998, chez Flammarion, sous le titre Liszt. Rhapsodie et improvisation ${ }^{4}$. Si l'essentiel des citations données dans cet article appartient au diptyque reconstitué (le livre de 1979 et la réédition de 1998), il convient de ne pas négliger deux autres ouvrages essentiels pour la connaissance de Jankélévitch en général et de la verve en particulier : le fameux La Musique et l'Ineffable, paru au Seuil en 1983 et, en 1978, chez Gallimard, un livre d'entretiens très rédigés, Quelque part dans l'inachevés. Tantôt dédicataire, tantôt interlocutrice du philosophe, Béatrice Berlowitz assure un lien amical entre ces deux dernières publications, comme tout à l'heure le travail de Françoise Schwab reconstituait le diptyque inachevé sur la virtuosité de Liszt. Fort de ces quatre ouvrages, on se propose dans cet article de suivre pas à pas les métamorphoses de la verve, de paraphraser (au sens musical) le cheminement subtil qui conduit de la virtuosité à l'ineffable. Cheminement subtil et aussi très étrange... Virtuosité, verve, ineffable : les trois termes ne donnent-ils pas le sentiment d'une grande disparité? Quand le premier, la virtuosité, tire la verve vers le bas, la ramenant à son niveau le plus superficiel, le second, l'ineffable, hausse le ton, mais au prix d'un paradoxe intenable, qui tente de concilier la verve du vaudevilliste et l'ineffable de Mélisande. C'est l'élucidation de ce paradoxe que l'on propose au lecteur - en suivant non pas la chronologie de l'œuvre de Jankélévitch, mais la logique secrète d'une pensée qui exalte l'état de verve comme essence de l'humanité.

3. Abrégé $L R$, suivi du numéro de la page.

4. Abrégé $L R I$, suivi du numéro de la page. En 1988, F. Schwab publie sous le titre La Musique et les Heures, les chap. II, III, IV, du livre de I955.

5. Abrégé $Q$, suivi du numéro de la page. 


\section{De la virtuosité à la verve}

Au commencement, donc, la virtuosité, que l'on définira comme le spectacle de la "difficulté vaincue» ( $L R, \mathrm{p}$. I8). Le livre de 1979, cherchant à rendre compte d'une telle attirance de manière équitable, fait se succéder un chapitre "pour» et un chapitre "contre» la virtuosité du pianiste et des musiciens. Le même souci de distance se retrouve dans le livre d'entretien avec Béatrice Berlowitz: «Les musclés, les forts, les "costauds", ceux que Max Jacob appelait les rugissants, auxquels il faut ajouter les cantatrices vociférantes, les virtuoses en délire et les chefs d'orchestre glorieux : ce camp triomphal n'est pas le nôtre.» (Q, p. 147) La charge est sans appel, Jankélévitch renouant ainsi avec les célèbres reproches qu'adressait Adorno à la "musique de chef d'orchestre", c'est-à-dire à toute la musique à effets et à l'opéra wagnérien, en particulier, obsédé par le geste au détriment de l'art. En s'en prenant à la virtuosité, Jankélévitch participe au discours antimusical qui apparaît de façon récurrente, de Platon à Quignard, chez les philosophes ou les écrivains. Le refrain est connu : c'est la force même de la musique qui en constitue la faiblesse, c'est sa puissance de séduction qui la transforme en une entreprise de pouvoir et de mort ("Qui sait? l'irréductible séducteur est peut-être le diable...», $L R$, p. $\left.5 \mathrm{I}^{6}\right)$. Liée à la «Burla ${ }^{7}$ " à l' « ivresse», la virtuosité de l'interprète a partie liée au mensonge, aux hallucinations collectives, aux grands sacrifices humains. C'est d'abord l'agacement devant l'affichage de tant de vacuité : "La musique des virtuoses parle sans avoir rien à dire, et elle est d'autant moins dense que le déchet sonore est plus encombrant." ( $L R$, p. 132) Puis la machine semble s'emballer toute seule, passer de la scène à la salle, soudée l'une et l'autre par une même complicité : "Les admirateurs, ayant acclamé la performance admirable, acclament ensuite l'acclamation, font une ovation à leurs propres ovations, applaudissent leurs propres applaudissements.» (LR, p. IO5) Et pour finir, c'est une mise à mort qui ne s'anoblit même pas de la violence initiatique du Sacre du printemps: «au bout du crescendo, il n'y a plus que le lynchage.» ( $L R$, p. I06)

Ce sévère constat s'explique-t-il seulement par le souci de consonner avec les critiques de son temps? Si proche d'Adorno ou précurseur du Quignard de La Haine de la musique, Jankélévitch joue sa propre partition et opte pour une double attitude à l'égard de la virtuosité. On hasardera un jugement

6. Le pouvoir du diable s'étend de la virtuosité à la verve : "C'est la verve du démon qui inspire au musicien ces éclats, comme elle lui inspire les dissonances, le chromatisme presque atonal, les accords altérés, les sublimes bizarreries de la Faust-Symphonie.» (LRI, p. 47)

7. «l'état de Burla. C'est une certaine recherche de l'étrange et du bizarre qui s'exprime tantôt dans la diablerie, tantôt dans la bouffonnerie» ( $L R$, p. 45). 
personnel, qui n'engage que l'auteur de ces lignes, même si certaines conversations particulières montrent que ce jugement est partagé : n'y a-t-il pas du virtuose en Jankélévitch et pas toujours de la meilleure veine? On peut le dire autrement : en chargeant la virtuosité, Jankélévitch ne règle-t-il pas quelques comptes avec lui-même et son propre penchant pour l'ivresse des mots et des concepts? Malgré l'admiration sincère que suscite une œuvre de haute tenue, le brio de l'écriture, l'emportement des mots (dont témoignent en particulier les entretiens si volubiles et si écrits à la fois) donnent parfois une impression de bavardage, de délayage, comme si la pensée se démultipliait sans contrôle, portée par la gourmandise des mots. En critiquant la virtuosité envisagée dans ses pires travers, Jankélévitch donne à sa sévérité une double fonction : il séduit son lecteur à force de lucidité et trouve à se distancier pour ne pas être dupe de lui-même. Dans un cas comme dans l'autre, l'opération, entre séduction et catharsis, rend à la virtuosité la vénération qu'elle mérite.

Comme Jankélévitch le reconnaît sans ambages : «Notre rapport avec le virtuose est ambivalent.» ( $Q$, p. 222) Mais l'ambivalence va peu à peu laisser place au naturel :

Aussi éprouvons-nous à la fois de l'admiration et de la rancune : admiration pour l'exploit, le tout de force que constituent le concerto et le récital, mais rancune aussi : nous nous en voulons à nous-mêmes, de notre propre admiration; peut-être le virtuose est-il un charlatan qui nous dupe, un acrobate qui nous mystifie? Nous admirons la prouesse et refusons la poudre aux yeux. (Q, p. 223)

Si le jugement semble reprendre la sévérité des condamnations antérieures, la suite immédiate prépare et accomplit un véritable coup de théâtre :

Cette ambivalence résume au fond nos rapports avec la musique : nous sommes subjugués par elle et nous lui en voulons d'être subjugués, les deux ensemble. D'autre part, la virtuosité est liée organiquement à la mythologie et à l'hagiographie du piano, à sa légende, à ses héros, à ses génies, à ses martyrs, à ses enfants prodiges; notre engouement pour le virtuose est justifié puisque nous rendons ainsi hommage au miracle que l'homme accomplit avec ses dix doigts. ( $Q$, p. 223)

La justification suit ainsi un double parcours. D’abord, Jankélévitch associe étroitement virtuosité et musique, l'une et l'autre liées par la même puissance de séduction. Quand tant de philosophes voient la virtuosité comme le ver dans le fruit (Adorno en tête sans doute), développant à l'égard de la musique une oreille soupçonneuse, Jankélévitch sauve la virtuosité en la rapprochant de la musique. D'un côté, la virtuosité compromet la musique, de l'autre la musique rédime la virtuosité. À cette première justification s'en ajoute une seconde, plus générale et plus philosophique, qui entraîne la virtuosité et la musique dans un humanisme plein d'exaltation. 
Malgré tous ses défauts, le virtuose se donne en effet pour l'emblème de la créativité, portée à son plus haut degré d'invention ("Le virtuose fait ", $L R$, p. 13). Plus que le chef d'orchestre partagé entre le théâtre et la fosse, c'est naturellement le pianiste qui incarne la virtuosité par excellence. Si l'organologie du XIX ${ }^{\mathrm{e}}$ siècle met à la disposition du musicien un instrument très sophistiqué, c'est la main qui vaut comme outil principal d'un homo faber survolté. Depuis l'entrée du chœur dans l'Antigone de Sophocle et son bel hommage à l'humanité capable de fendre la mer par la proue, le sol par la charrue et surtout capable d'ordonner le monde par la pensée, les pages de Jankélévitch sur la virtuosité constituent l'ensemble le plus exalté et le plus exaltant sur la grandeur de l'homme créateur. Comme il est dit à propos de Liszt : «Le virtuose démontre à l'homme tout ce que peut un hommes.» (LR, p. I3)

Mais pour que la main devienne pleinement créatrice, pour que la virtuosité se dépouille de sa brillance et révèle la verve au travail, il faut que l'interprète cède la place au compositeur; il faut passer de la simple exécution à une véritable création (qu'elle soit de notes ou de mots). S’il admire profondément le génie du pianiste qui sert la partition, quitte à se servir d'elle, Jankélévitch accorde l'essentiel de sa faveur au composteur ("dans le génie de Liszt le créateur et l'interprète ne font qu'un ", $L R$, p. I57). Ce trajet de l'exécution à la création, de la virtuosité à la verve passe par des étapes que l'on restituera d'une manière très synthétique. Entre l'instrumentiste et le compositeur, l'improvisation se présente comme un maillon essentiel :

elle désigne encore le mystère de la parturition mentale, elle est le commencement du commencement, la première démarche de l'invention créatrice à partir du rien de la feuille blanche, à partir de l'amorphe et de la parole balbutiante ( $Q$, p. 40).

Que l'on exalte le Liszt pianiste, le Liszt improvisateur ou le Liszt compositeur, c'est toujours la même puissance qui se montre à l'œuvre. Pour peu qu'on ne la réduise pas à une écume que le moindre vent dissipe, la virtuosité conduit à la verve grâce à la rigueur du travail et à la discipline de l'ascèse ( $\mathrm{N}^{\prime}$ 'y a-t-il pas quelque chose d'ascétique dans cette rigueur imposée à la prodigalité lisztienne?», $L R$, p. $\left.73^{9}\right)$

8. Jankélévitch règle implicitement ses comptes avec une grande partie de l'art contemporain, à commencer par l'art conceptuel : "La sincérité du virtuose se reconnaît à la performance effective et non pas à des discours sur la performance ni à des dissertations sur le concept de performance.» (LR, p. 42)

9. "Capricieux, brillant, fantasque, improvisateur... oui, mais comment deviner ce qui se cache sous ce halètement, dans cette parole fiévreuse, cette écriture emportée? Ce qui se cache sous cette apparence, c'est un travail méticuleux, presque maniaque. Je creuse mon sillon dans un monde fort étroit et je marche obstinément, soucieusement dans mes propres traces. J'envie les créateurs généreux, débraillés, insouciants, qui gaspillent tous leurs trésors et jettent 


\section{De la verve à l'ineffable}

Comment décrire l' «état de verve», expression qui revient régulièrement dans les ouvrages musicologiques de Jankélévitch? Soucieux de reconstituer un cheminement de pensée qui ne correspond à aucune chronologie d'écriture, on commencera par des œuvres pianistiques parmi les plus célèbres et les plus virtuoses :

Les Études «transcendantes» de Liszt et de Liapounov sont transcendantes en ceci qu'elles affirment la transcendance manuelle et digitale de l'homme sur la naturalité. Les réflexes gymnastiques, la résistance et l'inertie de la matière annulées, le corps absolument docile au dictamen du génie, l'homme enfin se jouant de la difficulté et jonglant avec les mondes, voilà où la virtuosité apparaît comme la forme technique de la verve. L'homme en verve et l'homme virtuose possèdent un excédent de pouvoirs, d'éloquence, de maîtrise instrumentale dont ils ne savent que faire et qu'ils dépensent en traits et vocalises, comme le rossignol. ( $L R I$, p. 92)

Tout signifie dans cette citation d'une remarquable densité : l'équivalence de la virtuosité et de la verve, le pouvoir de la main, les risques de bavardages aussi ; mais ce qui importe sans doute, c'est le jeu de mots que permet le titre de ces études pour piano. Au fond, tout est affaire de transcendance. Quand il s'agit, habituellement, de valoriser la verve, on ne manque jamais de la rapprocher de l'enthousiasme, c'est-à-dire de la parole du Dieu qui parle en moi ou qui me fait parler. D'une certaine manière, Jankélévitch réactive cette tradition en tirant la verve du côté de l'Inspiration. Mais qu'on ne s'y trompe pas : les études transcendantes de Liszt nous rappellent que cette transcendance n’a rien à faire avec quelque divinité que ce soit. Grâce à cette forme de transcendance, la verve ne quitte jamais un univers purement humain où seul l'homme est sujet de l'action, où seul l'homme est capable de se dépasser ("À l'infini la transcendance peut être transcendée», $L R, \mathrm{p}$. 55). Dans cet univers sans dieu, qui fait de l'homme un dieu à la fois orgueilleux et modeste - orgueilleux par le pouvoir, modeste par la conscience de ses limites -, la verve regarde plutôt vers le vitalisme de Bergson (dont Jankélévitch est un spécialiste reconnu). Anti-platonicienne, la verve ne se tourne jamais vers le ciel des Idées. Il faut passer par un virtuose comme Liszt («apothéose de l'apparence», $L R$, p. 47)

leurs idées, à tous les vents, par la fenêtre, Cette prodigalité est belle. Les musiciens que j'admire le plus, Liszt et Moussorgski, vivaient dans un désordre génial que des ressources vitales fabuleuses et littéralement inépuisables leur permettaient de maîtriser; détestables gestionnaires, magnifiquement négligents, ils continuaient à travailler dans l'indifférence la plus complète à l'égard de l'œuvre déjà faite.» (QPI, p. I6) 
ou un mondain de génie comme Gracian pour lire une réhabilitation aussi forte de l'apparence contre l'essence :

Ce qui n'est rien ne se voit pas. Gracian ${ }^{10}$ raisonne ainsi : Dieu a créé la splendeur resplendie avec la splendeur resplendissante, l'apparence avec l'essence; il faut donc prendre l'apparaître au sérieux. L'apparaître ne serait pas sans l'être. Le coucher du soleil, la rose et le paon sont les trois formes emblématiques de la splendeur. (Q, p. 226)

Cette assomption de l'immanence amène à reconsidérer la temporalité au bénéfice du présent. Le virtuose, l'improvisateur, et par extension l'homme en état de verve, non seulement vivent pleinement le présent ${ }^{\text {II }}$, mais comprennent que vivre et faire, c'est bander tous ces efforts pour donner forme au présent, sans céder à la contemplation délétère du passé (la nostalgie) ou de l'avenir (l'utopie). L'homme en état de verve, c'est tout simplement l'homme qui a réussi à trouver le kairos ${ }^{12}$ : l'improvisateur, le virtuose, l'homme d'esprit ${ }^{13}$ exemplifient cet art de l'à-propos dans lequel Jankélévitch voit le comble de l'humanité. Chez Liszt, c'est le mot "tact» qui vient insister sur la rencontre réussie de l'homme et du monde, ici et maintenant : «En fait, c'est affaire d'intuition, ou, mieux encore, de tact, le tact, qui est la divination de l'à-propos temporel, ajoute une nouvelle dimension au toucher virtuose.» ( $L R$, p. 93)

Ce sens de l'à-propos qui caractérise l'homme en état de verve impose, comme on l'a vu, une véritable ascèse. Il faut une discipline de fer pour jouer en concert une étude de Liszt, pour improviser à l'orgue sur un thème donné, pour affronter les exigences du présent sans céder aux sirènes de la mauvaise foi ou du rêve démobilisateur. En même temps, l'homme en état de verve découvre l'ivresse de sa liberté, comprend que le poids des contraintes trouve à s'équilibrer par l'invention de la forme, de cette forme singulière que l'on donnera à l'œuvre et plus généralement à sa vie. Ad libitum, a piacere : tels sont les mots d'ordre que découvre le virtuose, l'improvisateur de génie comme l'homme sans qualités. Choisissant de voir en Liszt ce héros que l'humanité gagnerait à se donner comme modèle, Jankélévitch n'oublie pas d'enraciner son humanisme, d'historiciser son approche philosophique et anthropologique. En s'intéressant à Liszt et à la rhapsodie, Jankélévitch regarde vers le XIX ${ }^{\mathrm{e}}$ siècle, les révolutions politiques et musicales qui caractérisent l'Europe centrale autour

Io. Gracian est le «virtuose de la rhétorique, de la métaphore et du calembour» ( $L R$, p. 45).

II. Un présent qui déborde à peine sur le passé ou sur l'avenir : «Selon qu'elle est antécédente ou conséquente, tension vers l'instant l'improvisation serait attente du futur en instance ou rétrospectivité minima.» (LRI, p. Io9)

I2. Sur le kaïros et le tact, voir $L R$, p. 82.

I3. «Bon mot, mot d'esprit ou trait d'esprit, le jeu de mot est en quelques sorte le pizzicato du discours" (LR, p. 92). 
de i848. Au vent de liberté dont témoigne le développement des nationalismes, correspond l'invention d'une forme nouvelle, cette rhapsodie qui permet au créateur d'échapper aux règles tout établies pour trouver son propre cheminement et modeler le présent. Héros de la verve, exemplum d'une humanité véritablement créatrice, Liszt triomphe avec une forme capable d'inventer sans cesse son propre développement, voire de rompre avec toute idée de développement, telle qu'on la trouve dans la symphonie ou la dissertation. Jankélévitch ne manque pas d'associer ces deux grandes structures qui habitent l'imaginaire d'un philosophe et d'un musicologue formés à l'école française ou allemande :

Nos symphonies, avec le débat de leurs thèmes, leur réexposition, leur cérémonial, sont en état de dissertation chronique; comme s'il avait des idées à approfondir et à expliciter, une thèse à prouver, comme s'il avait un sens et une téléologie, c'està-dire allait quelque part en se rapprochant de plus en plus de la conclusion, le discours musical prétend dérouler une dialectique rectiligne et continue : il obéit en cela à des analogies dialectico-rhétoriques qui assimilent la sonate au discours latin, au sermon et au plaidoyer. (LRI, p. 52-53)

Comment échapper à la dissertation ou à la symphonie? Cette question caractérise ce que l'on appelle communément la «modernité». Tout le $\mathrm{xx}^{\mathrm{e}}$ siècle, ou plutôt un certain $\mathrm{Xx}^{\mathrm{e}}$ siècle, valorise le fragment, le discontinu, le supplément, la dissémination. Dans son livre fameux, Les Fleurs de Tarbes, Paulhan a longuement déploré le soupçon généralisé jeté sur la «rhétorique» comme code collectif et modèle de construction (c'est la compositio qui constitue la principale cible). Très proche de Barthes sur ce point, malgré tout ce qui les sépare, Jankélévitch participe à cette remise en cause des structures établies, nouant avec le poststructuralisme des années 1970 un dialogue inattendu. Les faits sont toujours plus riches que les théories qui tentent de leur donner sens. Si «être moderne, c'est savoir ce qui n'est plus possible ${ }^{\mathrm{r}}{ }^{\text {» }}$, s'il convient d'opposer les créateurs qui s'inscrivent dans le mouvement de l'Histoire à tous ceux qui restent au bord du chemin ou regardent vers le passé, la discrimination ainsi établie peine à rendre compte de la complexité du réel. En ce début du XXI ${ }^{e}$ siècle, il est difficile de déterminer dans quelle direction va l'Histoire; un simple regard en arrière montre sans mal que la créativité a suivi simultanément des voies très différentes, que la remise en cause du récit (le Nouveau Roman) s'est combinée avec le développement de grands romans d'aventures (Giono, Perec), que la mort du sujet n'a pas détruit le souci de soi. De même, la frontière clairement dessinée entre les modernes et les conservateurs, la

I4. R. Barthes, «Réquichot et son corps», dans Euvres complètes, t. IV, éd. É. Marty, Paris, Seuil, 2003, p.397. 
périphérie (l’École pratique des hautes études ou Vincennes) et le centre de l'université française (la Sorbonne) ne sépare plus aussi nettement qu'on l'a cru des pratiques souvent habitées par les mêmes principes fondamentaux. Ainsi de Jankélévitch et de la philosophie contemporaine : professeur à la Sorbonne, musicologue reçu dans les salons parisiens, homme de l'Institution, bergsonien en plein marxisme, Jankélévitch n'a guère compté aux yeux d'un jeune lectorat qui regardait du côté de Foucauld, Derrida ou Deleuze. Et pourtant, entre, d'un côté, la "différance» ou le supplément derridiens, la signifiance ou la combinatoire ${ }^{15}$, l'enchaînement des métaphores ${ }^{16}$, chère à tant de poststructuralistes (Barthes, Kristeva), et, de l'autre, l'ineffable propre à Jankélévitch, les convergences sautent aux yeux dessillés, c'est-à-dire libérés des certitudes du «moderne».

Mais que faut-il entendre par «ineffable», ce mot qui accompagne la pensée de Jankélévitch au point de la résumer tout entière? Et quelle relation un mot comme «ineffable», associé la plupart du temps au monde des demi-teintes, aux non-dits de Maeterlinck ou à la poésie verlainienne, entretient-il avec la «verve» et ses feux d'artifice et, plus paradoxalement encore, avec la volatile écume de la "virtuosité"? La réponse est à lire dès l'ouverture de l'ouvrage majeur que Jankélévitch a consacré à la musique, La Musique et l'Ineffable. Cette fois-ci, le lecteur ne se voit plus confronté à la virtuosité, comme si la verve s'était assagie ou plutôt avait trouvé d'autres voies pour continuer à être elle-même :

le mystère que la musique nous transmet n'est pas l'inexprimable stérilisant de la mort, mais l'inexprimable fécond de la vie, de la liberté et de l'amour; plus brièvement : le mystère musical n'est pas l'indicible, mais l'ineffable. C'est la nuit noire de la mort qui est l'indicible, parce qu'elle est ténèbre impénétrable et désespérant non-être, et parce qu'un mur infranchissable nous barre de son mystère : est indicible, à cet égard, ce dont il n'y a absolument rien à dire, et qui rend l'homme muet en accablant sa raison et en médusant son discours. Et l'ineffable, tout à l'inverse, est inexprimable par ce qu'il y a sur lui infiniment, interminablement à dire : tel est l'insondable mystère de Dieu, tel l'inépuisable mystère d'amour, qui est mystère poétique par excellence [...] L'ineffable déclenche en l'homme l'état de verve. Sur l'ineffable il y a de quoi parler et chanter jusqu'à la consommation des siècles...» (Q, p. 92-93)

I5. «les fruits abondent en toute saison au verger des idées reçues, et la nouveauté n’est jamais que dans l'arrangement." (LRI, p. I23)

I6. "Aussi faudrait-il parler du temps comme Plotin parle de l'ineffable : recourant à une métaphore, pour ensuite la détruire, puis en trouver une autre plus légère, puis une autre encore, et finalement briser toutes les métaphores les unes contre les autres, et, à partir de leurs débris, suggérer une je-ne-sais-quoi qui est l'horizon de l'ineffable.»( $Q$, p. 29) 
Il sera temps bientôt de s'interroger longuement sur l'opposition de l'ineffable et de l'indicible, aussi violente que celle de la vie et de la mort. Mais pour l'instant, le lecteur peu familier de l'univers de Jankélévitch s'étonnera sans doute de voir le mot "verve» servir de transition entre la virtuosité de l'improvisateur et les mystères fauréens. Comment parler sans paradoxe intenable de la verve des "Nocturnes» pour piano ou du fameux Requiem? Les relais pourtant ne manquent pas : de manière générale, la verve correspond au plus haut sommet de la créativité humaine, au miracle du kairos, à la coïncidence euphorique du geste, de l'acte et de l'instant. Cet accomplissement de soi pour être exemplifié par le virtuose n'en concerne pas moins tous les modes d'expression, tous les styles et tous les tons, tous les types de créateurs. À ce titre donc, une berceuse de Fauré témoigne autant d'un état de verve que la Sonate en si de Liszt. Mais ce trait général ne suffit pas, à moins de confondre verve et créativité pour régler la question - et ne rien régler du tout.

En affirmant que «La Berceuse, c'est la chronométrie en débandade et le solfège en déliquescence...» ( $L R I$, p. I45), Jankélévitch définit un nouveau point commun entre la verve et l'ineffable. La liberté du cheminement, l'inventivité formelle qui se joue de tous les schémas imposés établit un lien secret entre les débordements du piano lisztien et la mélancolique retenue de l'art de Fauré, qui offre «le paradoxe d'une virtuosité discrète» ( $L R$, p. I59-I60) Le lecteur non averti acceptera-t-il de troquer un paradoxe au prix d'un élégant oxymore? On sortira d'embarras en rappelant une des qualités majeures de la verve et de la virtuosité : la profusion ${ }^{17}$. Dans une belle formule, Jankélévitch définissait ainsi le génie de l'homme pleinement accompli : «L'homme est un être limité qui n’a pas de limites indépassables; avec la limitation lui est donnée la possibilité de se transcender, sinon à l'infini du moins indéfiniment» $(L R$, p. 26-27) En jouant sur les mots («infini» et «indéfiniment»), Jankélévitch réussit à dessiner un monde sans Dieu, sans absolu, qui préfère le dynamisme de l'action à l'incommensurabilité de l'univers. Révélé par la virtuose qui en fait souvent trop, l'excès, la profusion constituent le cœur même de la verve. Si l'état de verve consiste naturellement à occuper le présent, à lui donner forme, à le dessiner à sa mesure, il s'agit surtout de prendre conscience de la prolifération comme son constituant majeur ("partout la surabondance est le corollaire de la virtuosité», $L R$, p. I8). Cette surabondance, c'est le geste de celui qui en fait trop, mais c'est aussi la lucidité de celui qui accepte l'ineffable comme emportement non mesuré de la parole et du sens. Il suffit une fois encore de retenir les leçons de l'improvisation :

I7. «Il est, cet homme en verve, le génie biologique des métamorphoses» (LRI, p. I05); «la virtuosité est le pouvoir d'être autre que soi, de devenir son propre contraire» (LR, p. 62) 
Tout est dit? Non, rien n'est dit. Ou plutôt tout est à dire et même si tout était dit, tout serait encore "à redire", comme s'exprime Liszt lui-même; et la "grande année» des combinaisons musicales est située à l'infini dans l'avenir pour une imagination qui puise dans l'infini du passé et qui s'échappe toujours à elle-même. (LRI, p. I48-I49)

Tout à l'heure, Jankélévitch mettait sur le même plan, l'homme en verve, l'homme virtuose et le rossignol, qui dépensent tous les trois leur "excédent de pouvoirs" "en traits et vocalises». Une plus juste appréhension de l'ineffable conduit Jankélévitch à mieux différencier les rôles, à hiérarchiser les hommes et l'oiseau :

Soit un géomètre, un rhapsode et un rossignol perchés sur la même branche. Le rossignol ne fait rien d'autre que ses vaines vocalises. Le géomètre, lui, ne chante pas des arpèges, pas plus que le Code civil n'implique des trilles et des roulades. Entre le rossignol et le géomètre, l'homme-rossignol, c'est-à-dire le poète, s'offre le luxe de la vocalise pour suggérer indirectement un sens - car la poésie obéit à d'autres lois que le principe utilitaire d'économie. ( $L R I$, p. I42)

Cette confrontation sur la branche rassemble, sous le mot de "poète», les pratiques les plus diverses : le pianiste improvisateur qui subjugue le public ici et maintenant, le compositeur qui a écrit la partition, le créateur de mots ou de sons qui approchent le mystère du monde sans jamais mettre un point final à son élucidation, le critique ou le philosophe qui ajoutera ses mots à d'autres mots avant que d'autres mots soient "indéfiniment» ajoutés au déjà de la culture. L'ineffable, c'est à la fois la richesse inépuisable du monde que l'artiste en verve restitue au plus près et le commentaire sans fin que suscite sa représentation : il y a sans cesse à dire sur la nuit que dépeint Fauré et sur le Nocturne qui a donné forme à sa verve musicale. Parti de l'interprète, remonté jusqu'au compositeur et à tout créateur, Jankélévitch conduit par une virtuosité qui cherche à tout embrasser en revient à l'interprète, à cet homme des mots qui analysent les partitions et pratiquent une herméneutique sans fin.

\section{De l'ineffable à l'innommable}

«La virtuosité enfin n'est pas liée à l'indicible : elle implique au contraire la furie de l'expression.» ( $L R$, p. 9) : cette expression furieuse, pleine de verve, qui coïncide avec l'ineffable, se définit comme valeur par opposition à une contrevaleur, l'indicible, que Jankélévitch prive du prestige que revêt souvent le mot. Quel sens donner à cet antonyme dont la fonction n' est pas seulement de créer la signification par antithèse? À quelles réalités, que l'on devine inquiétantes, 
renvoie-t-il ? Dans la longue citation tirée de La Musique et l'Ineffable, le mot donne lieu à une approche indécise, métaphorique, comme s'il s'agissait de rester discret ou de signaler la difficulté évidente de dire l'indicible. Les autres textes de Jankélévitch se montrent beaucoup plus explicites. Et le lecteur, peutêtre étourdi par tant de verve, d'enthousiasme humaniste, comprend peu à peu que cet indicible renvoie largement à la tragédie dont les juifs ont été les victimes pendant le Seconde guerre mondiale.

Dans ses ouvrages musicologiques, quand Jankélévitch s'attarde avec ivresse sur Liszt, la virtuosité et l'improvisation, le monde juif n'apparait jamais directement. C'est sur le mode de l'allusion que l'on relève des développements qu'on lira peu à peu comme des messages cryptés. Ainsi de ce passage sur les Tziganes, qui par l'errance manifestent leur verve créatrice : «Plus heureux que la gueuserie même, les nomades de la musique ne possèdent rien, et pas même un folklore; ils ne thésaurisent pas les thèmes; ils inventent au fur et à mesure ce qu'ils chantent et ils l'oublient ensuite; ils sont le prolétariat dans le prolétariat...» (LRI, p. 38) Mais la comparaison qui suit immédiatement propose un signe qu'il n’est pas difficile de déchiffrer : «Si les camps de concentration avaient été inventés, M. Guizot et la philosophie du bas de laine les y eussent enfermés.» (LRI, p. 38) Il faudra attendre un livre d'entretiens pour que Jankélévitch s'explique plus directement. Et face à Béatrice Berlowitz, les propos sont limpides :

En fait l'extermination de six millions de juifs est l'invisible mauvaise conscience de toute la modernité : elle pèse comme un accablant secret sur tous nos contemporains, qu'ils en aient ou non conscience et même s'ils n'en éprouvent aucun remords. C'est le terrifiant, l'indicible secret que chacun porte plus ou moins en soi.» (Q, p. 67)

Se tisse alors dans toute la vie et l'œuvre de Jankélévitch un réseau très complexe d'ineffable et d'indicible, de parole et de silence, de révélation et de secret. Devant le souvenir de l'innommable, il est nécessaire à la fois de se taire à jamais et de parler sans arrêt : de se taire, parce qu'il n'y a rien à dire sur les camps et la Shoah et parce que l'événement déroute toutes les significations, et, en même temps, de continuer à parler, de chercher l'état de verve qui donnera forme au présent et réconciliera l'homme avec lui-même.

Par son refus de la culture allemande après 1945, par une pensée organisée autour de l'ineffable, Jankélévitch répond à sa manière au défi que la Seconde Guerre mondiale lance à la philosophie et à la musique. Chacun répond comme il peut, c'est-à-dire comme il pense : Jankélévitch ne propose par la même réponse qu'Adorno, auquel il se réfère ici ou là, et sa réponse ne sera pas celle non plus de philosophes comme Deleuze, Badiou ou Zizek. Au fond, la 
question est double : dans quelle mesure Auschwitz vaut-il comme une rupture dans l'Histoire (un peu comme la Révolution française pour Michelet)? et que peut la parole poétique (au sens le plus large du terme) face à un tel événement? C'est à cette double injonction que les philosophies de la musique ont été sommées de répondre, en revenant une fois encore sur le "cas Wagner».

Si tout le monde s'accorde pour condamner l'horreur des camps nazis, le statut de cette tragédie ne fait pas l'unanimité. Peu à peu, la pensée d'Adorno s'est imposée dans l'opinion à partir des années 1960 comme la lecture de référence. Selon le philosophe allemand, Auschwitz se présente comme un événement d'une singularité absolu, c'est-à-dire comme un événement qui ne peut être assimilé à aucun autre dans l'Histoire. Sur ce point, Jankélévitch et Béatrice Berlowitz développent des analyses très voisines :

Considérer l'antisémitisme comme un racisme parmi d'autres permet d'intégrer plus sereinement Auschwitz à l'Histoire. Or la persécution des juifs ne peut être assimilée à aucune autre persécution - et il ne suffit pas de dire qu'elle les dépasse toutes en horreur; encore faut-il analyser les modalités d'une pratique sans précédent. (Q, p. I38)

À cette analyse dominante de nos jours encore, Alain Badiou et Slovej Zizek opposent une approche sensiblement différente. Dans ses Cinq leçons sur le "cas» Wagner", Alain Badiou consacre la seconde de ses cinq leçons à «La dialectique négative d'Adorno ", livre où il est à peine question de Wagner, mais qui donne la clé de la relation que le philosophe allemand entretient avec le compositeur. S'éloignant de la musique pour mieux y revenir, Badiou analyse pas à pas la pensée d'Adorno confrontée au scandale que représente Auschwitz dans l'histoire de l'humanité. Décrits comme une rupture fondamentale, comme le comble de la barbarie, les camps nazis correspondent au degré le plus élevé d'aliénation que la dialectique impose à l'altérité. Poursuivant son compte rendu, Badiou confronte Dialectique négative à l'Essai sur Wagner pour montrer comment Adorno inscrit les opéras de Wagner dans la dynamique tragique de la métaphysique allemande. Comme on s'en doute, Wagner n'est jamais donné comme le responsable des camps; mais sa participation très active au mythe politique de la culture allemande, la prédominance chez lui du principe d'identité (mélodie infinie, soumission de la musique au théâtre...), s'inscrivent dans cette évolution qui conduit à la catastrophe. Manifestement, Badiou comme Zizek récusent cette analyse qui sort Auschwitz de l'Histoire et hypothèque l'avenir ou fait dépendre toute analyse politique de l'énigme et du scandale de la Shoah. Sans trop entrer dans le débat, on peut se demander

I8. A. Badiou, Cinq leçons sur le «cas» Wagner, Caen, Nous, 20IO; Slavoj Zizek, Variations Wagner, Caen, Nous, 2010. 
si Jankélévitch n'apporte pas malgré qu'il en ait un argument de poids à ces dernières analyses. D'un côté, il affirme, comme Adorno, la singularité absolue du génocide, mais d'un autre côté sa pensée de l'instant semble aller à l'encontre de cette première affirmation. En effet, "Chaque moment de notre vie n'advient qu'une seule fois dans toute l'éternité et ne sera plus jamais.» ( $L R$, p. 7$)$ Dans ces conditions, c'est chaque instant qui est affirmé dans sa singularité. Est-il alors vraiment possible de penser une singularité plus singulière que les autres? S'il est concevable de distinguer voire de hiérarchiser les horreurs, comment peut-on détacher une singularité sans remettre en cause la singularité des autres événements?

On comprend dès lors la grande différence dans l'usage de la parole. Bien connue est la terrible injonction qu'Adorno tire de l'Histoire; c'est d'abord l'impossibilité de toute poésie après Auschwitz (c'est-à-dire de toute forme d'art); puis c'est un infléchissement de cette position vers une attitude compassionnelle, la poésie étant appelée à témoigner contre la barbarie. Ainsi, les camps nazis, coupant l'histoire en deux et donnant forme au devenir de l'humanité, définiraient les devoirs de l'artiste comme de l'intellectuel. Le discours très critique sur Wagner témoigne d'une parole philosophique et politique qui se donne pour fonction d'accuser les responsables et de rendre justice aux victimes. À l'évidence, Alain Badiou et Slovej Zizek choisissent un tout autre usage de la parole. Pour sauver Wagner, pour entendre le final de Parsifal ou du Crépuscule des dieux comme l'esquisse d'une nouvelle communauté humaine et une relance possible de l'Histoire, il faut s'opposer à la lecture réductrice d'Adorno. Comme on s'en doute, il ne s'agit pas pour les deux philosophes marxistes de nier ou de sous-estimer l'horreur des camps et du génocide; il s'agit, grâce à Wagner ou avec lui, de rompre avec la sacralisation du génocide que symbolise le mot «Shoah» et à laquelle la pensée d'Adorno a si brillamment concouru. En relativisant le génocide sans pour autant en rabattre sur sa spécificité et sur son horreur, ils refusent de penser l'Histoire comme coupée en deux par Auschwitz et de limiter la parole à la réparation.

Le cas de Jankélévitch est sensiblement différent ${ }^{19}$. Quand Adorno accuse Wagner, quand Alain Badiou et Slovej Zizek le réhabilitent, Jankélévitch choisit le silence pur et simple. Après 1945, comme on le sait, il renonce à la culture allemande, quel que soit le prix à payer quand on est philosophe et

19. Jankélévitch partage une grande partie les analyses d'Adorno : "Il y a un mot d'Adorno qui peut-être désigne toute le détresse de la modernité : "Après Hegel et Auschwitz»... Votre cuvre, notre vie depuis 1945 semblent entièrement tournées par cette présence de l'innommable et par votre volonté de ne pas monnayer l'horreur en valeurs pensables. Pour moi qui ai eu la chance inouïe, inestimable de n'avoir pas été à Auschwitz, et dont les parents n’ont pas été déportés, c'est un devoir sacré de témoigner. Inlassablement.» $(Q$, p. 67) 
musicologue. Cette attitude qui impose le respect, même si son outrance ne convainc pas, correspond à un premier usage - paradoxal - de la parole : le silence dénonciateur, le refus du dialogue, l'impossible pardon. Mais cette position ne correspond que très partiellement au philosophe de la verve et de la virtuosité. Plutôt que de développer une parole accusatrice ou même réparatrice comme le pratique Adorno, Jankélévitch préfere remettre le logos en marche. Toute son œuvre philosophique et musicologique témoigne que la vie doit continuer, que l'homme n'est pas mort et qu'il lui appartient de retrouver sa dignité offusquée par l'Histoire. À mi-chemin d'Adorno qui parle pour réparer, d'Alain Badiou qui parle pour continuer à penser le monde politiquement, Jankélévitch subordonne sa parole à Auschwitz, mais pour n'en pas parler directement. La meilleure réponse possible à la barbarie, c'est de retrouver l'état de verve, c'est de parier sur l'optimisme fondamental qui anime la musique. Mais comme on s'en doute, le chemin est escarpé : «Entre la distance glaciale et la brûlante proximité, où et comment assigner l'insaisissable optimisme ${ }^{20}$ ?» $(L R$, p. 77$)$

20. "Comme il y a un scepticisme dans l'attente d'un dogmatisme, ainsi il y a un pessimisme provisoire qui est prélude à l'optimisme : voilà la grande différence entre le pessimisme fin-de-siècle que systématisera Schopenhauer avec sa métaphysique du malheur, et le pessimisme début-de-siècle des romantiques.» $(L R, \mathrm{p} .72)$ 